\title{
Association between insomnia and coping style in Japanese patients with type 2 diabetes mellitus
}

This article was published in the following Dove Press journal:

Neuropsychiatric Disease and Treatment

\author{
Kazutaka Yoshida' \\ Hideyuki Otaka ${ }^{2}$ \\ Hiroshi Murakami² \\ Hirofumi Nakayama' \\ Masaya Murabayashi \\ Satoru Mizushiri² \\ Koki Matsumura ${ }^{2}$ \\ Jutaro Tanabe 2 \\ Yuki Matsuhashi \\ Miyuki Yanagimachi ${ }^{2}$ \\ Norio Sugawara ${ }^{3}$ \\ Kazuhiko Nakamura' \\ Makoto Daimon ${ }^{2}$ \\ Norio Yasui-Furukori' \\ 'Department of Neuropsychiatry, \\ Hirosaki University Graduate \\ School of Medicine, Hirosaki, Japan; \\ ${ }^{2}$ Department of Endocrinology and \\ Metabolism, Hirosaki University \\ Graduate School of Medicine, \\ Hirosaki, Japan; ${ }^{3}$ Department of \\ Clinical Epidemiology, Translational \\ Medical Center, National Center of \\ Neurology and Psychiatry, Tokyo, \\ Japan
}

Correspondence: Norio Yasui-Furukori Department of Neuropsychiatry, Hirosaki University Graduate School of Medicine,

Zaifu-cho 5, Hirosaki 0.36-8562, Japan

Tel +8I I72 395066

Fax +8I I72 395067

Email yasufuru@hirosaki-u.ac.jp
Purpose: Insomnia, which is associated with type 2 diabetes mellitus (DM), results in a low quality of life, and several relationships exist between insomnia and coping style. Thus, we clarified the association between some coping styles and insomnia among Japanese type 2 DM patients.

Subjects and methods: The subjects included 503 type 2 DM patients (mean age $63.9 \pm 12.5$ years). Sleep disturbance and personality traits were evaluated using the Japanese version of the Pittsburgh Sleep Quality Index and the Brief Scale for Coping Profile, respectively. Lifestyle factors, glycated hemoglobin A1c (HbA1c) levels, and the depression statuses of the patients were also included in the analyses.

Results: Among the 503 subjects with type 2 DM, 141 (28.0\%) subjects exhibited probable insomnia. After adjusting for confounders, being female, living alone, and using "avoidance and suppression" were significantly correlated with current insomnia. No other relationships were found between insomnia and $\mathrm{HbA} 1 \mathrm{c}$ or lifestyle factors, such as smoking, drinking alcohol, and exercise frequency.

Conclusion: The prevalence of insomnia in individuals with type 2 DM was high, and the protective factors included some emotion-focused coping styles. Future prospective studies are required to confirm the therapeutic effects of behavioral interventions on insomnia in patients with type 2 DM.

Keywords: cross-sectional studies, coping style, Japanese, insomnia, type 2 DM

\section{Introduction}

The global costs of diabetes and its consequences are large and will substantially increase by $2030 .{ }^{1}$ Asia is a major area for the rapidly emerging type 2 diabetes mellitus (DM) global epidemic, with China and India being the top two epicenters. Although genetic predisposition partially determines individual susceptibility to type $2 \mathrm{DM}$, an unhealthy diet and a sedentary lifestyle are important drivers of the current global epidemic; early developmental factors (such as intrauterine exposure) also play a role in the susceptibility to type $2 \mathrm{DM}$ later in life. Most patients with type $2 \mathrm{DM}$ have at least one complication, and cardiovascular complications are the leading cause of morbidity and mortality in these patients. ${ }^{2}$ Thus, the high prevalence of type $2 \mathrm{DM}$ as a lifestyle-related disease has become a further burden and type $2 \mathrm{DM}$ can increase the risk of serious physical and mental health issues.

Sleep disturbance, particularly insomnia (defined as trouble initiating or maintaining sleep), is one of the most frequent mental disorders ${ }^{3,4}$ leading to clinically relevant impairments in health-related quality of life. ${ }^{5}$ In recent years, several studies have indicated that short or disturbed sleep is associated with glucose intolerance, insulin resistance, reduced acute insulin response to glucose, ${ }^{6,7}$ and increased risks for developing 
type 2 diabetes. ${ }^{8-11}$ Over $40 \%$ of type 2 DM patients suffer from insomnia, and the prevalence of insomnia is higher in type $2 \mathrm{DM}$ patients than in the general population. ${ }^{12-15}$

Coping is regarded as a mediator of the relationship between stress and illness. ${ }^{16,17}$ Effective coping styles that involve actively engaging problems, positively interpreting situations, and using humor lead to faster resolution of difficulties and help maintain the psychological health of an individual during times of stress, thereby allowing a greater sense of safety and security. By contrast, ineffective coping styles lead to poorer psychological health and allow one to avoid problematic situations and vent feelings; these coping styles do not lead to a resolution of difficulties and may lead to an exacerbation of problems, thereby disturbing sleep. ${ }^{18,19}$

We hypothesized that insomnia is associated with coping style, which may be linked to glucose control, although there is little information on the relationship between insomnia and coping strategies. Therefore, we examined the association between insomnia and coping style in type $2 \mathrm{DM}$ patients.

\section{Subjects and methods Subjects}

The current study was approved by the ethics committee of the Hirosaki University Graduate School of Medicine, and all subjects provided written informed consent before participating in this study.

This study included 728 individuals with type 2 DM who received treatment for at least 1 year at the Department of Endocrinology and Metabolism at Hirosaki University Hospital. We recruited 945 patients, and 728 patients agreed to participate in the survey. The remaining 217 patients were excluded because of a decline (41 patients), moderate-to-severe dementia (85 patients), blindness (23 patients), or moderateto-severe psychiatric diseases (eg, bipolar disorder and schizophrenia; 68 patients).

A total of 611 patients of the 728 patients returned the questionnaires, and 503 (69.1\%) patients had complete questionnaires. Blood samples from these patients were collected routinely for glycated hemoglobin A1c (HbA1c) analysis at least four times per year. Among the subjects, 408 patients were receiving an oral hypoglycemic agent and 235 patients were receiving insulin therapy. The demographic data (age, sex, smoking history, alcohol consumption, solitary living, and exercise habits) and medical histories of the patients were obtained from the questionnaires and medical records.

Subjective sleep difficulty was assessed using the validated Japanese version of the Pittsburgh Sleep Quality
Index (PSQI-J) ${ }^{20,21}$ The PSQI-J is a self-rated questionnaire that measures sleep difficulty, including sleep disturbance, retrospectively for a 1-month period, with total scores ranging from 0 to 21. Increased PSQI-J scores indicate greater sleep difficulty. Insomnia was defined as 5.5 or above on the PSQI-J.

We used the Brief Scale for Coping Profile (BSCP) to assess coping behaviors. The BSCP consists of 18 items rated on a 4-point scale. ${ }^{22,23}$ The participants were asked to indicate the frequency, which ranged from 1 (almost never) to 4 (very often), at which they used the strategy described by a particular item in stressful situations. The scale assesses an individual's ability to cope with daily stressful circumstances using the following six subscales: "active solution (AC)", "seeking help for a solution (S)", "changing mood (CM)", "emotional expression involving others (EE)", "avoidance and suppression (AV)", and "changing one's point of view (CV)".

The Japanese version of the Center for Epidemiological Studies Depression (CES-D) scale was used for all patients to measure depressive symptoms. ${ }^{24,25}$ The CES-D is a 20-item, self-report measure that focuses on depressive symptoms during the week prior to the administration of the questionnaire. The maximum score on this scale is 60 , and a CES-D score of $\geq 16$ is indicative of the presence of depression.

\section{Data analyses}

Herein, comparisons of several factors between insomnia and noninsomnia were performed using Student's $t$-tests and chi-square tests. The data are presented as the mean $\pm \mathrm{SD}$. A $P$-value of $<0.05$ indicated statistical significance. The factors associated with insomnia, including age, sex, body mass index (BMI), HbA1c, smoking status, alcohol consumption, solitary living, exercise frequency, and score for each of the six coping subscales, were examined using forward stepwise logistical regression analyses. In addition, regression analyses were adjusted for the confounding factors. Linear regression analyses with forward stepwise selection were performed to examine the correlation between the severity of insomnia and several factors. The dummy variables were as follows: male $=1$, female $=2$, presence of spouse $=1$, absence of spouse $=2$, living with family $=0$, living alone $=1$, presence of smoking $=1$, absence of smoking $=2$, presence of alcohol consumption $=1$, absence of alcohol consumption $=2$, no exercise $=1$, exercise once a week $=2$, exercise $2-3$ days per week $=3$, exercise $4-5$ days per week $=4$, and exercise almost every day $=5$. A $P$-value of $<0.05$ indicated statistical significance. The IBM SPSS Statistics software program 
for Windows Version 24.0 (IBM Corporation, Armonk, NY, USA) was used for all analyses.

\section{Results}

A total of 141 (28.0\%) of the 503 patients had current sleep disturbances/insomnia based on the PSQI-J cutoff value. The sex, age, BMI, solitary living, depression, and use of "avoidance and suppression" factors differed significantly between patients with and without current insomnia, but the HbA1c, lifestyle, and marital status factors did not (Table 1). The HbA1c levels did not differ between the patients with and without insomnia.

Because strong colinearities existed between the CES-D score and coping style, the CES-D score was removed from the multiple variable regression. Table 2 shows the results of the logistical regression analysis that were used to determine the factors associated with sleep disturbances/insomnia, such as being female, BMI, and use of avoidance and suppression. Multiple regression analysis showed the same results, indicating that using avoidance and suppression, using "changing one's point of view", living alone, being female, and being young were correlated with the severity of insomnia as measured by the total PSQI-J score (Table 3). Multiple regression analyses did not reveal a correlation between the HbA1c level and insomnia severity.

\section{Discussion}

The present study investigates the prevalence of probable insomnia as measured by the PSQI-J and assessed the relationship between insomnia and coping styles among individuals

Table I Characteristics of subjects with and without insomnia

\begin{tabular}{|c|c|c|c|}
\hline Characteristics & $\begin{array}{l}\text { With insomnia } \\
(n=141)\end{array}$ & $\begin{array}{l}\text { Without insomnia } \\
(n=362)\end{array}$ & Significance \\
\hline \multicolumn{4}{|l|}{ Sex } \\
\hline Male, n (\%) & $68(48)$ & $224(62)$ & $P<0.0$ I \\
\hline Female, n (\%) & $73(52)$ & $138(38)$ & \\
\hline Age (years) & $61.3 \pm 13.6$ & $64.9 \pm 11.9$ & $P<0.01$ \\
\hline BMI & $26.1 \pm 4.7$ & $24.7 \pm 4.0$ & $P<0.01$ \\
\hline HbAlc (\%) & $7.1 \pm 1.0$ & $7.1 \pm 0.8$ & ns \\
\hline \multicolumn{4}{|l|}{ Smoking habit } \\
\hline Yes, n (\%) & $24(17)$ & $55(15)$ & ns \\
\hline No, n (\%) & 117 (83) & $307(85)$ & \\
\hline \multicolumn{4}{|l|}{ Habitual alcohol consumption } \\
\hline Yes, n (\%) & $39(28)$ & $121(33)$ & ns \\
\hline No, n (\%) & $102(72)$ & $24 \mid(67)$ & \\
\hline \multicolumn{4}{|l|}{ Exercise frequency } \\
\hline No, n (\%) & $87(62)$ & $\mid 86(5 \mid)$ & \\
\hline Once a week, n (\%) & $10(7)$ & $22(6)$ & \\
\hline 2-3 times a week, n (\%) & $22(16)$ & $52(14)$ & ns \\
\hline 4-5 times a week, n (\%) & $12(9)$ & $35(10)$ & \\
\hline Almost every day, n (\%) & $19(13)$ & $67(18)$ & \\
\hline \multicolumn{4}{|l|}{ Single } \\
\hline Yes, n (\%) & $51(36)$ & $99(27)$ & ns \\
\hline No, n (\%) & $90(64)$ & $263(73)$ & \\
\hline \multicolumn{4}{|l|}{ Living alone } \\
\hline Yes, n (\%) & $25(18)$ & $38(10)$ & $P<0.05$ \\
\hline No, n (\%) & $116(82)$ & $324(90)$ & \\
\hline Depression (CES-D) & $17 . \mid \pm 8.7$ & $11.6 \pm 6.8$ & $P<0.00$ I \\
\hline \multicolumn{4}{|l|}{ Coping profile } \\
\hline Active solution & $6.3 \pm 2.5$ & $6.2 \pm 2.7$ & ns \\
\hline Seeking help for a solution & $8.1 \pm 2.7$ & $8.0 \pm 2.9$ & ns \\
\hline Changing mood & $7.8 \pm 2.6$ & $8.2 \pm 2.6$ & ns \\
\hline Emotional expression involving others & $10.9 \pm 1.7$ & $11.2 \pm 1.5$ & ns \\
\hline Avoidance and suppression & $9.1 \pm 2.2$ & $9.5 \pm 2.1$ & $P<0.05$ \\
\hline Changing one's point of view & $7.8 \pm 2.4$ & $7.5 \pm 2.8$ & ns \\
\hline
\end{tabular}

Notes: Data are shown as mean \pm SD. Current insomnia is defined as $\geq 5.5$ on the PSQI-J.

Abbreviations: BMI, body mass index; CES-D, Center for Epidemiological Studies Depression; HbAIc, hemoglobin Alc; ns, no significance; PSQI-J, Japanese version of the Pittsburgh Sleep Quality Index. 
Table 2 Logistic regression results for factors associated with insomnia among type 2 DM patients

\begin{tabular}{|c|c|c|c|c|}
\hline Variables & Wald & Significance & $\operatorname{Exp}(\beta)$ & $95 \% \mathrm{Cl}$ \\
\hline Sex & 6.19 & 0.013 & 1.68 & I.12-2.52 \\
\hline \multicolumn{5}{|l|}{ Age } \\
\hline BMI & 8.01 & 0.005 & 1.07 & $1.02-1.12$ \\
\hline \multicolumn{5}{|l|}{$\mathrm{HbAlc}$} \\
\hline \multicolumn{5}{|l|}{ Smoking } \\
\hline \multicolumn{5}{|l|}{ Alcohol } \\
\hline \multicolumn{5}{|l|}{ Exercise frequency } \\
\hline \multicolumn{5}{|l|}{ Single } \\
\hline Living alone & 5.11 & 0.024 & 1.93 & $1.09-3.40$ \\
\hline \multicolumn{5}{|l|}{ Coping profile } \\
\hline \multicolumn{5}{|l|}{ Active solution } \\
\hline \multicolumn{5}{|l|}{ Seeking help for a } \\
\hline \multicolumn{5}{|l|}{ solution } \\
\hline \multicolumn{5}{|l|}{ Changing mood } \\
\hline \multicolumn{5}{|l|}{ Emotional expression } \\
\hline \multicolumn{5}{|l|}{ involving others } \\
\hline $\begin{array}{l}\text { Avoidance and } \\
\text { suppression }\end{array}$ & 6.13 & 0.013 & 0.88 & $0.80-0.97$ \\
\hline $\begin{array}{l}\text { Changing one's point } \\
\text { of view }\end{array}$ & 3.91 & 0.048 & 1.09 & $1.11-1.19$ \\
\hline
\end{tabular}

Note: Current insomnia is defined as $\geq 5.5$ on the PSQI-J.

Abbreviations: BMI, body mass index; DM, diabetes mellitus; HbAlc, hemoglobin Alc; PSQI-J, Japanese version of the Pittsburgh Sleep Quality Index.

with type 2 DM using a cross-sectional study design. This study is the first to identify a significant association between the absence of insomnia and the use of avoidance and suppression, an inverse association between the severity of insomnia and the use of avoidance and suppression, and a possible association between the severity of insomnia and the use of changing one's point of view in individuals with type $2 \mathrm{DM}$. The six BSCP subscales reflect the following three different coping dimensions: problem-focused ( $\mathrm{AC}$ and $\mathrm{S}$ ), adaptive emotion-focused (CM and EE), and maladaptive emotionfocused (AV and CV) coping strategies. ${ }^{22,23}$ Despite being in the same category, avoidance and suppression and changing one's point of view were oppositely associated with insomnia. Avoidance and suppression was protective, and changing one's point of view was a risk factor for insomnia.

Insomnia occurs in $>90 \%$ of patients with clinical depression. As a symptom, insomnia has a $60 \%-70 \%$ positive predictive value in diagnosing major depressive disorder (MDD). More than $50 \%$ of patients presenting to sleep disorder clinics have insomnia, and those who present with insomnia have a higher prevalence of MDD. The coping profile is a mediator that influences mood, ${ }^{26}$ and we therefore removed the CES-D score from our regression analysis because too high of a correlation was found between the CES-D score and the PSQI-J score.
Table 3 Simple and multiple regression results for factors associated with the severity of insomnia among type 2 DM patients

\begin{tabular}{lllll}
\hline Variables & $\boldsymbol{r}$ & Significance & $\beta$ & Significance \\
\hline Sex & 0.129 & $\mathbf{0 . 0 0 2}$ & $0.11 \mathrm{I}$ & $\mathbf{0 . 0 1 2}$ \\
Age & -0.098 & $\mathbf{0 . 0 1 4}$ & -0.097 & $\mathbf{0 . 0 2 9}$ \\
BMI & 0.100 & $\mathbf{0 . 0 1 3}$ & & \\
HbAlc & 0.024 & 0.296 & & \\
Smoking & -0.045 & 0.159 & & \\
Alcohol & 0.014 & 0.379 & & \\
Exercise frequency & -0.028 & 0.265 & & \\
Single & 0.134 & $\mathbf{0 . 0 0 1}$ & & \\
Living alone & 0.142 & $\mathbf{0 . 0 0 1}$ & 0.151 & $<\mathbf{0 . 0 0 0}$ \\
Coping profile & & & & \\
$\quad \begin{array}{l}\text { Active solution } \\
\text { Seeking help for a }\end{array}$ & -0.017 & 0.057 & & \\
solution & 0.022 & 0.315 & & \\
$\begin{array}{l}\text { Changing mood } \\
\text { Emotional expression }\end{array}$ & -0.043 & 0.170 & & \\
involving others & -0.074 & $\mathbf{0 . 0 4 8}$ & & \\
$\begin{array}{l}\text { Avoidance and } \\
\text { suppression } \\
\text { Changing one's point } \\
\text { of view }\end{array}$ & -0.104 & $\mathbf{0 . 0 1 0}$ & -0.111 & $\mathbf{0 . 0 1 3}$ \\
$\begin{array}{l}\text { Multiple correlation } \\
\text { coefficients }\end{array}$ & & 0.057 & 0.112 & $\mathbf{0 . 0 1 3}$ \\
\hline
\end{tabular}

Notes: Current insomnia is defined as $\geq 5.5$ on the PSQI-J. Bold indicates significance. Abbreviations: BMI, body mass index; DM, diabetes mellitus; HbAlc, hemoglobin Alc; PSQI-J, Japanese version of the Pittsburgh Sleep Quality Index.

Our results were inconsistent with the hypothesis that participants who typically use disengagement coping have worse sleep based on several key indicators of sleep health. Avoidance coping, however, has been identified as an effective short-term strategy. A functional coping style is considered protective, while a nonfunctional coping style is considered a risk factor for depression. Similar to avoidance and suppression, denial is used in "an attempt to reject the reality of the stressful event." ${ }^{27}$ In the long term, however, avoidance coping impedes psychological adjustment and increases distress symptoms, such as depression. ${ }^{28-30}$ Emotion-focused coping has been described as the strongest mediator of the stress-illness relationship. Stress-related illness, deterioration in general health status, and poor physical health have been found to occur more frequently in those who consistently employ emotion-focused coping. ${ }^{19}$ Morin et $\mathrm{al}^{31}$ demonstrated that emotion-focused coping is associated with increased perceived stress. Such strategies are assumed to be more common in the insomnia population. In addition, Sadeh et $\mathrm{al}^{32}$ showed that for the group of students with good sleep, high emotion-focused coping scores are more likely to correspond to a decrease in total sleep time during the week prior to an important interview. Fernandez-Mendoza et $\mathrm{l}^{33}$ indicated that compared to people who are not vulnerable, vulnerable people are more likely to 
employ maladaptive coping strategies and to report increased emotional cognitive arousal. For young offenders, increased emotion-focused coping predicted increased anxiety and insomnia $(P<0.0001)$, whereas increased rational coping predicted decreased symptoms $(P<0.005)$. For juveniles, increased emotion-focused coping predicted increased anxiety and insomnia $(P<0.0001) .{ }^{34}$

Interestingly, the scores for avoidance and suppression and "emotional expression involving others", which are regarded as negative coping styles, were higher in one study's subjects than in a healthy population in Japan. ${ }^{35}$ Values for Japanese references and subjects in this study were 6.6 and 9.4 for avoidance and suppression and 4.4 and 7.6 for emotional expression involving others, respectively, although a direct comparison was not conducted. ${ }^{23}$ Therefore, using coping strategies such as maladaptive emotion-focused coping may be one method for treating multiple chronic disorders.

A previous review found no effect of stress on sleep parameters in adults. ${ }^{36}$ In the subset of nine studies that used measures of daily stress and stressful life events, the results were mixed, but the best evidence indicates that stress affects sleep continuity. In addition, the sleep duration measured by actigraphy was unrelated to the measured daily hassles. ${ }^{37}$

Several studies reported no correlations between insomnia and $\mathrm{HbA} 1 \mathrm{c}$ in patients with type $2 \mathrm{DM},{ }^{38,39}$ while increased $\mathrm{HbA1c}$ was significantly related to insomnia in a Japanese male subclinical population. ${ }^{40}$ Thus, the association between $\mathrm{HbA1c}$ values and sleep disturbance is still inconclusive. A previous study showed that the treatment of sleep problems in diabetic patients did not lead to a reduction in $\mathrm{HbA} 1 \mathrm{c} .^{38}$ Therefore, HbA1c may not be a predictive factor for insomnia in patients with type $2 \mathrm{DM}$. A recent review showed that sleep quality was significantly related to glycemic control in 14 of 22 studies, while nine studies found no relationship between any measure of sleep and glycemic control. ${ }^{41}$ Compared to normal sleep, short and long sleep durations were associated with increased $\mathrm{HbA1c}$, suggesting a U-shaped dose-response relationship. Similarly, poor sleep quality was associated with increased HbAlc. ${ }^{41}$

Several systematic reviews have found that short sleep duration is associated with a greater risk of obesity, ${ }^{42,43}$ while a recent meta-regression analysis showed that the association between sleep and obesity is still inconclusive. ${ }^{44}$ People with obesity may have hypoventilation syndrome or obstructive sleep apnea (OSA), which are both related to sleep disorders, ${ }^{45}$ possibly affecting the results of our study.

Several studies have suggested that living alone is correlated with long-term insomnia. ${ }^{39}$ Thus, these results suggest that living alone may be a cause of insomnia even in this type $2 \mathrm{DM}$ population. In addition, the results of this study suggest that being female is a risk factor for insomnia. Several previous studies have shown a higher prevalence of insomnia in women than in men, ${ }^{46-48}$ while a meta-analysis showed no significant associations between the prevalence of insomnia and sex. ${ }^{49}$ Therefore, the evidence for an effect of sex on the prevalence of insomnia is still inconclusive.

This study has several limitations. First, due to the lack of a control group, a reference score for the neuroticism personality trait could not be provided. Studies that include a control group are needed. Second, since this study was a cross-sectional study, identifying a causal relationship between the presence and severity of insomnia and risk factors was difficult. Third, all patients who had subjective insomnia in this study were not clinically diagnosed. We defined insomnia based on the PSQI scores, which measure only sleep quality and do not cover all the diagnostic criteria needed to diagnose insomnia according to the International Classification of Sleep Disorders. Objective sleep measures are necessary to make a definite diagnosis and clarify the presence/absence of other sleep disorders that are potentially causative of the participants' subjective disturbances in sleep maintenance, such as OSA and restless legs syndrome. Finally, since the subjects were recruited from a single institute, hidden sampling bias may be present in this study. Therefore, a large sample from multiple centers is required to confirm our findings.

\section{Conclusion}

The risk factors were being female and living alone, and the protective factor was using the avoidance and suppression coping style. Future prospective studies are required to confirm the therapeutic effects of behavioral interventions for insomnia in patients with type 2 DM.

\section{Acknowledgments}

This study was funded by a Grant-in-Aid for Scientific Research (KAKENHI) from the Japan Society for the Promotion of Research (JSPS, 15K19239, 15K19710, $15 \mathrm{H} 04754$, and $15 \mathrm{~K} 01643$ ). The funders had no role in the study design, data collection and analysis, decision to publish, or preparation of the article.

\section{Disclosure}

NY-F received grant/research support or honoraria from and has been a speaker for Dainippon-Sumitomo Pharmaceutical, Mochida Pharmaceutical, and MSD. The authors report no other conflicts of interest in this work. 


\section{References}

1. Bommer C, Sagalova V, Heesemann E, et al. Global economic burden of diabetes in adults: projections from 2015 to 2030. Diabetes Care. 2018;41(5):963-970.

2. Zheng Y, Ley SH, Hu FB. Global aetiology and epidemiology of type 2 diabetes mellitus and its complications. Nat Rev Endocrinol. 2018;14(2):88-98. doi: 10.1038/nrendo.2017.151.

3. Morin CM, Drake C, Harvey AG, et al. Insomnia disorder. Nat Rev Dis Primers. 2015;1:15026.

4. Riemann D, Nissen C, Palagini L, Otte A, Perlis ML, Spiegelhalder K. The neurobiology, investigation, and treatment of chronic insomnia. Lancet Neurol. 2015;14(5):547-558.

5. Kyle SD, Morgan K, Espie CA. Insomnia and health-related quality of life. Sleep Med Rev. 2010;14:69-82.

6. Buxton OM, Pavlova M, Reid EW, Wang W, Simonson DC, Adler GK. Sleep restriction for 1 week reduces insulin sensitivity in healthy men. Diabetes. 2010;59(9):2126-2133.

7. Schmid SM, Hallschmid M, Jauch-Chara K, Bandorf N, Born J, Schultes B. Sleep loss alters basal metabolic hormone secretion and modulates the dynamic counterregulatory response to hypoglycemia. J Clin Endocrinol Metab. 2007;92(8):3044-3051.

8. Beihl DA, Liese AD, Haffner SM. Sleep duration as a risk factor for incident type 2 diabetes in a multiethnic cohort. Ann Epidemiol. 2009; 19(5):351-357.

9. Chao CY, Wu JS, Yang YC, et al. Sleep duration is a potential risk factor for newly diagnosed type 2 diabetes mellitus. Metabolism. 2011;60(6): 799-804.

10. Chaput JP, Després JP, Bouchard C, Astrup A, Tremblay A. Sleep duration as a risk factor for the development of type 2 diabetes or impaired glucose tolerance: analyses of the Quebec Family Study. Sleep Med. 2009;10(8):919-924.

11. Tuomilehto H, Peltonen M, Partinen M, et al. Sleep duration, lifestyle intervention, and incidence of type 2 diabetes in impaired glucose tolerance: the Finnish Diabetes Prevention Study. Diabetes Care. 2009; 32(11):1965-1971.

12. Vgontzas AN,LiaoD,PejovicS,CalhounS,KaratarakiM,BixlerEO.Insomnia with objective short sleep duration is associated with type 2 diabetes: a population-based study. Diabetes Care. 2009;32(11):1980-1985.

13. Cappuccio FP, D’Elia L, Strazzullo P, Miller MA. Quantity and quality of sleep and incidence of type 2 diabetes: a systematic review and meta-analysis. Diabetes Care. 2010;33(2):414-420.

14. Anothaisintawee T, Reutrakul S, Van Cauter E, Thakkinstian A. Sleep disturbances compared to traditional risk factors for diabetes development: systematic review and meta-analysis. Sleep Med Rev. 2016;30:11-24.

15. Vgontzas AN, Tan TL, Bixler EO, Martin LF, Shubert D, Kales A. Sleep apnea and sleep disruption in obese patients. Arch Intern Med. 1994;154(15):1705-1711.

16. Kamann MP, Wong BY. Inducing adaptive coping self-statements in children with learning disabilities through self-instruction training. J Learn Disabil. 1993;26(9):630-638.

17. Carver CS, Pozo C, Harris SD, et al. How coping mediates the effect of optimism on distress: a study of women with early stage breast cancer. J Pers Soc Psychol. 1993;65(2):375-390.

18. Taylor SE, Stanton AL. Coping resources, coping processes, and mental health. Annu Rev Clin Psychol. 2007;3:377-401.

19. Matthews KA, Hall MH, Cousins J, Lee L. Getting a good night's sleep in adolescence: do strategies for coping with stress matter? Behav Sleep Med. 2016;14(4):367-377.

20. Buysse DJ, Reynolds CF 3rd, Monk TH, Berman SR, Kupfer DJ. The Pittsburgh Sleep Quality Index: a new instrument for psychiatric practice and research. Psychiatry Res. 1989;28(2):193-213.

21. Doi Y, Minowa M, Uchiyama M, et al. Psychometric assessment of subjective sleep quality using the Japanese version of the Pittsburgh Sleep Quality Index (PSQI-J) in psychiatric disordered and control subjects. Psychiatry Res. 2000;97(2-3):165-172.
22. Kageyama T, Kobayashi T, Kawashima M, Kanamaru Y. Development of the Brief Scales for Coping Profile (BSCP) for workers: basic information about its reliability and validity. Sangyo Eiseigaku Zasshi. 2004;46(4):103-114. [In Japanese, Abstract in English].

23. Tomotsune Y, Sasahara S, Umeda T, et al. The association of sense of coherence and coping profile with stress among research park city workers in Japan. Ind Health. 2009;47(6):664-672.

24. Radloff LS. The CES-D scale: a self-report depression scale for research in the general population. Appl Psychol Meas. 1977;1:385-401.

25. Sugawara N, Yasui-Furukori N, Takahashi I, Matsuzaka M, Nakaji S. Age and gender differences in the factor structure of the Center for Epidemiological Studies Depression Scale among Japanese working individuals. Compr Psychiatry. 2015;56:272-278.

26. Khurshid KA. Comorbid insomnia and psychiatric disorders: an update. Innov Clin Neurosci. 2018;15(3-4):28-32.

27. Burker EJ, Evon DM, Losielle MM, Finkel JB, Mill MR. Coping predicts depression and disability in heart transplant candidates. J Psychosom Res. 2005;59(4):215-222.

28. Rhode P, Lewinsohn PM, Tilson M, Seeley JR. Dimensionality of coping and its relation to depression. J Pers Soc Psychol. 1990;58(3): 499-511.

29. Bryant RA, Harvey AG. Avoidant coping style and post-traumatic stress following motor vehicle accidents. Behav Res Ther. 1995;33(6): 631-635.

30. Holoha CJ, Moos RH, Holohan CK, Brennab PL. Social support, coping and depressive symptoms in a late middle-aged sample of patients reporting cardiac illness. Health Psychol. 1995;14(2):152-163.

31. Morin CM, Rodrigue S, Ivers H. Role of stress, arousal, and coping skills in primary insomnia. Psychosom Med. 2003;65(2):259-267.

32. Sadeh A, Keinan G, Daon K. Effects of stress on sleep: the moderating role of coping style. Health Psychol. 2004;23(5):542-545.

33. Fernandez-Mendoza J, Calhoun SL, Bixler EO, et al. Sleep misperception and chronic insomnia in the general population: role of objective sleep duration and psychological profiles. Psychosom Med. 2011;73(1): 88-97.

34. Ireland JL, Boustead R, Ireland CA. Coping style and psychological health among adolescent prisoners: a study of young and juvenile offenders. J Adolesc. 2005;28(3):411-423.

35. Sugawara N, Sato K, Takahashi I, et al. Depressive symptoms and coping behaviors among individuals with irritable bowel syndrome in Japan. Intern Med. 2017;56(5):493-498.

36. Kim EJ, Dimsdale JE. The effect of psychosocial stress on sleep: a review of polysomnographic evidence. Behav Sleep Med. 2007;5(4):256-278.

37. Hanson MD, Chen E. Daily stress, cortisol, and sleep: the moderating role of childhood psychosocial environments. Health Psychol. 2010; 29(4):394-402.

38. Garfinkel D, Zorin M, Wainstein J, Matas Z, Laudon M, Zisapel N. Efficacy and safety of prolonged-release melatonin in insomnia patients with diabetes: a randomized, double-blind, crossover study. Diabetes Metab Syndr Obes. 2011;4:307-313.

39. Nakajima H, Kaneita Y, Yokoyama E, et al. Association between sleep duration and hemoglobin A1c level. Sleep Med. 2008;9:745-752.

40. Kachi Y, Nakao M, Takeuchi T, Yano E. Association between insomnia symptoms and hemoglobin Alc level in Japanese Men. PLoS One. 2011;6:e21420.

41. Lee SWH, Ng KY, Chin WK. The impact of sleep amount and sleep quality on glycemic control in type 2 diabetes: a systematic review and meta-analysis. Sleep Med Rev. 2017;31:91-101.

42. Wu YL, ZShai L, Zhang DF. Sleep duration and obesity among adults: a meta-analysis of prospective studies. Sleep Med. 2014;15(12): 1456-1462.

43. Sperry SD, Scully ID, Gramzow RH, Jorgensen RS. Sleep duration and waist circumference in adults: a meta-analysis. Sleep. 2015;38(8): 1269-1276.

44. Chan WS, Levsen MP, McCrae CS. A meta-analysis of associations between obesity and insomnia diagnosis and symptoms. Sleep Med Rev. Epub 2017 Dec 15. 
45. Liu C, Chen MS, Yu H. The relationship between obstructive sleep apnea and obesity hypoventilation syndrome: a systematic review and meta-analysis. Oncotarget. 2017;8(54):93168-93178.

46. Krystal AD. Insomnia in women. Clin Cornerstone. 2003;5(3): 41-50.

47. Ohayon MM, Roth T. Place of chronic insomnia in the course of depressive and anxiety disorders. J Psychiatr Res. 2003;37(1):9-15.
48. Sivertsen B, Krokstad S, Overland S, Mykletun A. The epidemiology of insomnia: associations with physical and mental health. The HUNT-2 study. J Psychosom Res. 2009;67(2):109-116.

49. Cao XL, Wang SB, Zhong BL, et al. The prevalence of insomnia in the general population in China: a meta-analysis. PLoS One. 2017; 12(2):e0170772.

\section{Publish your work in this journal}

Neuropsychiatric Disease and Treatment is an international, peerreviewed journal of clinical therapeutics and pharmacology focusing on concise rapid reporting of clinical or pre-clinical studies on a range of neuropsychiatric and neurological disorders. This journa is indexed on PubMed Central, the 'PsycINFO' database and CAS, and is the official journal of The International Neuropsychiatric Association (INA). The manuscript management system is completely online and includes a very quick and fair peer-review system, which is all easy to use. Visit http://www.dovepress.com/testimonials.php to read real quotes from published authors.

Submit your manuscript here: http://www.dovepress.com/neuropsychiatric-disease-and-treatment-journal 